partial renal remission, or poor renal outcome, including chronic kidney disease (CKD) or end stage renal disease (ESRD).

Results: 499 patients were included ( $85.6 \%$ females) with a median follow-up of 10.6 years (IQR $4-18$ ). We observed an increase in both age at diagnosis of LN (P1 28.4 \pm 10.4 ; P2 29 \pm 11.5 ; P3 34.4 \pm 13.3 years) and disease duration before LN diagnosis (P1 1.3 \pm 1.3 ; P2 2.6 \pm 4.5 ; P3 4.6 \pm 6.3 years) from 1970 to 2016 ( $p<0.001$ for both). At clinical presentation, renal insufficiency and acute nephritic syndrome became less common (P1 14.2\%; P2 3.9\% ; P3 3.4\% and P1 29\%, P2 20.3\%; P3 $12.4 \%$, respectively, $\mathrm{p}<0.0001$ ) while isolated urinary abnormalities became significantly more prevalent from P1 to P3 (P1 26.4\%; P2 38\%; P3 48.9\%; $\mathrm{p}<0.0001$ ). Outcome was available in $95.8 \%$ of patients. Frequency of partial and complete renal remission progressively increased (P1 6.9\%; P2 28\%; P3 32\% and $\mathrm{P} 149.6 \%$; $\mathrm{P} 248 \%$; $\mathrm{P} 358.5 \% ; \mathrm{p}<0.001$ and $\mathrm{p}=0.01$, respectively) while $C K D$, ESRD and death decreased (P1 7.9\%; P2 8.5\%; P3 4.5\%; P1 24.8\% P2 9\%; P3 1.3\%; P1 19.8\%; P2 5.9\%; P3 3.6\%, respectively. $p<0.001$ for all). Survival without ESRD at 10 and at 20 years was $87 \%$ and $80 \%$ in $\mathrm{P} 1,94 \%$ and $90 \%$ in $\mathrm{P} 2 \%$ and $99 \%$ in P3 ( $p=0.0019)$. Induction therapy with immunosuppressants was more frequently performed over time (P1 71\%; P2 82\%; P3 94.6\%, p<0.0001) and use of MMF significantly increased both as induction and maintenance treatment (P1 0, P2 2.7\%; P3. 33.8\% and P1 1\%; P2 15\%; P3 54.8\%, respectively; $\mathrm{p}<0.0001)$. At multivariate analysis, logarithm of serum creatinine (RR:2.72), male gender (RR:3.34), activity index (RR:1.1), chronicity index (RR:1.29), arterial hypertension (RR:5.95), and lack of maintenance immunosuppressive therapy (RR:3.04) predicted ESRD. No significant changes in histological classes or active lesions at the time of renal biopsy were observed, while chronicity index significantly decreased from $\mathrm{P} 1$ to $\mathrm{P} 3(\mathrm{p}=0.023)$.

Conclusions: The clinical presentation of LN apparently became less severe in the last decades, likely due to earlier diagnosis and proper treatment, leading to an improved renal survival.

Disclosure of Interest: None declared

DOI: 10.1136/annrheumdis-2018-eular.7035

\section{FRI0375 DELAYED LUPUS NEPHRITIS IN THE COURSE OF SYSTEMIC LUPUS ERYTHEMATOSUS PREDICTS A POORER RENAL RESPONSE TO INDUCTION THERAPY, RENAL FLARES, AND WORSE LONG-TERM RENAL OUTCOMES: A MULTICENTER, RETROSPECTIVE COHORT STUDY}

M. Nakano ${ }^{1,2}$, K. Kubo $^{1}$, Y. Shirota ${ }^{3}$, Y. Iwasaki ${ }^{1}$, N. Tanaka ${ }^{2}$, Y. Takahashi ${ }^{2}$,

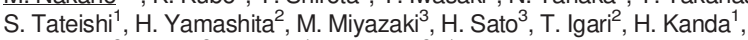
H. Kaneko ${ }^{2}$, T. Ishii ${ }^{3}$, K. Fujio ${ }^{1}$, A. Mimori ${ }^{2}{ }^{1}$ The University of Tokyo Hospital; ${ }^{2}$ National Center for Global Health and Medicine, Tokyo; ${ }^{3}$ Tohoku University Hospital, Sendai, Japan

Background: Some prognostic factors for lupus nephritis (LN) have been mentioned such as nephrotic syndrome, class 4 and chronicity on histology. In a previous single-centre study, we reported a potentially poorer renal response to induction therapy in LN that developed later after SLE onset (delayed, D-LN) compared with LN manifesting at SLE onset (early, E-LN) ${ }^{1}$ ). However, our study was limited by a small sample size and lack of long-term observation.

Objectives: This multicenter study aimed to validate whether D-LN was a useful predictor of a poor response to induction therapy with more detailed clinical and pathological parameters. Furthermore, we investigated whether D-LN was also a predictor of flares and long-term renal outcomes in addition to the established prognostic factors.

Methods: We retrospectively examined 215 biopsy-proven LN patients (136 ELN, 79 D-LN) who attended 3 hospitals above between 1997 and 2014 and who were observed for 3-20 (median: 10.8) years from LN onset. We compared baseline clinical, pathological features and treatment options at LN onset between E$\mathrm{LN}$ and D-LN. Then we compared the cumulative complete response (CR) rates, renal/extra-renal relapse rates, and the rates of renal insufficiency between the two groups. Renal insufficiency was defined as follows: ${ }^{1}$ serum creatinine (SCr) doubling or ESRD for severe insufficiency, and ${ }^{2} \mathrm{SCr}$ increasing by 1.5 times and also $>1.0$ (female) or $>1.2 \mathrm{mg} / \mathrm{dl}$ (male) for mild insufficiency. Moreover, we evaluated predictors of the response, flares and long-term renal outcomes with multivariate analysis.

Results: Anti-Sm/RNP antibodies and mixed proliferative and membranous nephritis (class $3+5$ or $4+5$ ) were significantly more prevalent in D-LN than E-LN ( 48.1 vs. $32.4 \%, 68.8$ vs. $40.4 \%, 40.5$ vs. $18.4 \%$, respectively). Log-rank test showed that significantly lower cumulative CR rates over 3 years $(65.8$ vs. $83.1 \%$ at 12 months, 83.5 vs. $92.6 \%$ at 36 months, $p<0.01)$ and significantly higher relapse rates (both renal and extra-renal) over 20 years in D-LN than E-LN. Mild renal insufficiency was significantly more likely to occur in D-LN over 20 years (21.5 vs. $12.5 \%, p=0.04$ ). We performed multivariate Cox regression analysis for the response, flare, and renal outcomes including significant variables on univariate analysis. As independent predictors of CR, D-LN, nephrotic syndrome and chronicity index were identified. As those of renal flares, D-LN was detected.
Although D-LN was not associated with severe renal insufficiency, D-LN was identified as an independent predictor of mild renal insufficiency as well as some other factors (table 1 )

\begin{tabular}{|c|c|c|c|c|c|}
\hline \multirow{2}{*}{\multicolumn{6}{|c|}{ Predictors of CR }} \\
\hline & & & & & \\
\hline $\mathrm{D}-\mathrm{LN}$ & $0.66(0.49-0.89)$ & 0.006 & $\mathrm{D}-\mathrm{LN}$ & $0.50(0.36-0.69)$ & k0.001 \\
\hline Serum creatinine & $0.61(0.46-0.81)$ & 0.001 & & & \\
\hline Nephrotic syndrome & $0.54(0.40-0.73)$ & $<0.001$ & Nephrotic syndrome & $0.51(0.37-0.70)$ & $<0.001$ \\
\hline Class IV & $0.66(0.49-0.87)$ & 0.004 & & & \\
\hline Activity Index & $0.94(0.90-0.97)$ & 0.001 & & & \\
\hline Chronicity index & $0.75(0.66-0.84)$ & $k 0.001$ & Chronicity index & $0.81(0.70-0.92)$ & 0.002 \\
\hline \multicolumn{6}{|c|}{ Predictors of renal flare } \\
\hline $\mathrm{D}-\mathrm{LN}$ & $2.50(1.55-4.03)$ & $<0.001$ & D-LN & $2.17(1.30-3.65)$ & 0.003 \\
\hline Anti-RNP antibodies & $1.71(1.05-2.78)$ & 0.030 & & & \\
\hline $\begin{array}{l}\text { Non-CR at } 12 \text { months } \\
\text { after induction therapy }\end{array}$ & 2.0 & 0.005 & & & \\
\hline \multicolumn{6}{|c|}{ Predictors of extra-renal flare } \\
\hline $\mathrm{D}-\mathrm{LN}$ & $1.92(1.05-3.52)$ & 0.035 & D-LN & $1.64(0.88-3.05)$ & 0.122 \\
\hline Anti-Sm antibodies & $2.49(1.34-4.65)$ & 0.004 & Anti-Sm antibodies & $2.31(1.23-4.34)$ & 0.009 \\
\hline \multicolumn{6}{|c|}{ Predictors of severe renal insufficiency } \\
\hline $\mathrm{D}-\mathrm{LN}$ & $2.31(0.89-5.99)$ & 0.085 & & & \\
\hline Anti-RNP antibodies & $9.41(2.14-41.3)$ & 0.003 & Anti-RNP antibodies & $10.1(2.27-44.6)$ & 0.002 \\
\hline Chronicity index & $1.42(1.03-1.97)$ & 0.032 & & & \\
\hline $\begin{array}{l}\text { Non-CR at } 6 \text { months } \\
\text { ffter induction therapy }\end{array}$ & $4.38(1.5$ & k0.001 & $\begin{array}{l}\text { Non-CR at } 6 \text { months } \\
\text { after induction therapy }\end{array}$ & $3.21(1.06-9.75)$ & 0.039 \\
\hline \multicolumn{6}{|c|}{ Predictors of mild renal insufficiency } \\
\hline $\mathrm{D}-\mathrm{LN}$ & $2.00(1.02-3.92)$ & 0.044 & D-LN & $2.12(1.03-4.34)$ & 0.041 \\
\hline Male & $2.14(1.02-4.48)$ & 0.044 & Male & $2.87(1.31-6.27)$ & 0.008 \\
\hline Serum creatinine & $1.97(1.45-2.67)$ & $<0.001$ & Serum creatinine & $1.97(1.30-2.99)$ & 0.002 \\
\hline Nephrotic syndrome & $2.48(1.26-4.88)$ & 0.009 & & & \\
\hline Activity index & $1.14(1.04-1.24)$ & 0.003 & & & \\
\hline Chronicity index & $1.77(1.42-2.19)$ & $<0.001$ & Chronicity index & $1.61(1.25-2.09)$ & $<0.001$ \\
\hline $\begin{array}{l}\text { Non-CR at } 6 \text { months } \\
\text { after induction therapy }\end{array}$ & $5.24(2.50-11.0)$ & $<0.001$ & $\begin{array}{l}\text { Non-CR at } 6 \text { months } \\
\text { after induction therapy }\end{array}$ & $3.29(1.46-7.4$ & 0.004 \\
\hline
\end{tabular}

Conclusions: D-LN might be a novel predictor of a poorer treatment response, renal flares and long-term renal outcomes independent of the established prognostic factors. The distinct differences in the autoantibody profiles between E-LN and D-LN groups suggest that D-LN patients might reflect a refractory SLE subset with a specific immunological profile.

\section{REFERENCE:}

[1] Nakano M, et al. Different responses to induction therapy in two onset categories of lupus nephritis. EULAR 2017 THU 0251

Acknowledgements: None

Disclosure of Interest: None declared

DOI: 10.1136/annrheumdis-2018-eular.4665

\section{FRI0376 DYSLIPIDEMIA AS A NEWLY RECOGNISEDFACTOR ASSOCIATED WITH DAMAGE ACCRUAL IN EARLY DIAGNOSED SLE: RESULTS FROM THE MULTICENTER EARLY LUPUS PROJECT INCEPTION COHORT}

M. Piga ${ }^{1}$, A. Zanetti ${ }^{2}$, G. Carrara ${ }^{2}$, C.A. Scirè ${ }^{2}$, I. Prevete $^{3}$, G.D. Sebastiani ${ }^{3}$, L. Coladonato ${ }^{4}$, F. lannone ${ }^{4}$, A. Bortoluzzi ${ }^{5}$, M. Govoni ${ }^{5}$, C. Tani ${ }^{6}$, M. Mosca $^{6}$, L. laccarino ${ }^{7}$, A. Doria ${ }^{7}$, M. Fredi ${ }^{8}$, A. Tincani ${ }^{8}$, F.R. Spinelli ${ }^{9}$, F. Conti $^{9}$, F. Bellisai ${ }^{10}$ M. Galeazzi ${ }^{10}$, A. Mathieu ${ }^{1} .{ }^{1}$ Università di Cagliari, Cagliari, ${ }^{2}$ Italian Society for Rheumatology, Milano; ${ }^{3}$ Ospedale San Camillo, Roma; ${ }^{4}$ Università di Bari, Bari; ${ }^{5}$ Università di Ferrara, Ferrara; ${ }^{6}$ Università di Pisa, Pisa; ${ }^{7}$ Università di Padova, Padova; ${ }^{8}$ Università di Brescia, Brescia; ${ }^{9}$ Università Sapienza, Roma; ${ }^{10}$ Università di Siena, Siena, Italy

Background: Preventing organ damage is a major challenge in Systemic Lupus Erythematosus (SLE).

Objectives: To evaluate factors associated with development of damage in a prospectively followed cohort of early diagnosed SLE patients.

Methods: The Early Lupus Project ${ }^{1}$ encompasses 9 Italian centres recruiting, from the 1 st January 2012, an inception cohort of consecutive patients diagnosed with SLE within 12 months from appearance of four or more 1997 ACR classification criteria. At study entry and then every 6 months a large panel of data was recorded.

Here, we report on factors associated with the development of damage assessed by the SLICC/ACR Damage Index (SDI). Using univariate analysis, we assessed the contribution of covariates collected at baseline (demographic, comorbidities serological, clinical by BILAG2004 domains, disease activity by ECLAM, HRQoL by visual analogic scale) in the development of damage (SDI from 0 to $>1$ ). Forward-Backward Cox-regression models were fitted with covariates with $p<0.05$ to identify factors independently associated with increased risk of damage development.

Results: Overall, 279 patients were enrolled in the Early Lupus Project inception cohort up to the 31 th of December $2017 ; 230$ patients (89.6\% Caucasians, $13.4 \%$ males) were eligible for this study having $\mathrm{SDI}=0$ at enrolment and at least 6 